\title{
Race for the Kerr field
}

\section{G. Dautcourt}

Received: 25 July 2008 / Accepted: 30 September 2008 / Published online: 18 October 2008 (C) The Author(s) 2008. This article is published with open access at Springerlink.com

\begin{abstract}
Roy P. Kerr has discovered his celebrated metric 45 years ago, yet the problem to find a generalization of the Schwarzschild metric for a rotating mass was faced much earlier. Lense and Thirring, Bach, Andress, Akeley, Lewis, van Stockum and others have tried to solve it or to find an approximative solution at least. In particular Achilles Papapetrou, from 1952 to 1961 in Berlin, was interested in an exact solution. He directed the author in the late autumn of 1959 to work on the problem. Why did these pre-Kerr attempts fail? Comments based on personal reminiscences and old notes.
\end{abstract}

Keywords Exact solutions - Axisymmetric stationary fields · Kerr metric · Black Hole $\cdot$ History of physics

\section{Introduction}

The old Prussian Academy of Sciences in Berlin was certainly a good place after the Second World War to continue the research of its most prominent former member, Albert Einstein. Here Einstein had worked 19 years and created his beautiful theory of gravitation. Here several attempts had been undertaken to test the theory: Einstein's young coworker, the astronomer Erwin Finley Freundlich, had tried to see (with little success) gravitational redshift effects in astronomical objects like the Sun. Freundlich was also active in several campaigns to observe Solar eclipses with the aim to verify the predicted displacement of stars seen near the Sun. And here Karl Schwarzschild, the director of the Astrophysical Observatory at Potsdam, a town at the outskirts of

G. Dautcourt $(\varangle)$

Max-Planck-Institut für Gravitationsphysik, Albert-Einstein-Institut,

Am Mühlenberg 1, 14476 Golm, Germany

e-mail: daut@aei.mpg.de 
Berlin, had published in 1916 the first exact solution of Einstein's field equations, describing the exterior gravitational field of a nonrotating spherical mass [44]. In view of the extreme nonlinearity of the equations it appeared almost as a miracle that exact solutions exist at all.

The administrative buildings of the Academy (in the summer of 1946 reopened as "German Academy of Sciences") and of the Berlin university happened to be situated in the Eastern part of the divided city of Berlin, thus the Academy worked under the influence of the Soviet Military Administration and later the East German government. Other scientific institutions such as institutes of the former Kaiser-Wilhelm society in Berlin-Dahlem and the 1948 founded Free University, also there, did belong to West Berlin, the sphere of influence of the Western Allies. The borders between the two parts of Berlin were open until 1961, allowing at least some personal contact between scientists of both sides. The director of the Academy since November 1946 was Josef Naas, a mathematician and member of the communist party, who was sent to a concentration camp in the time of the Nazi regime. He and other officials of the Academy were interested in a continuation of research on Einstein's path at this traditional place. In 1952 Achilles Papapetrou, a Greek scientist at the Physics Department of the University of Manchester, was invited as Senior Researcher to the Academy's Research Institute for Mathematics, headed by Naas. Papapetrou had started his academic career in solid state theory in the German town Stuttgart (thus he had a fluent knowledge of German), but he was known in the scientific community for his excellent work in relativity as well. The astrophysicists of the Academy planned further tests of Einstein's theory by astronomical means. Walter Grotrian, who now headed the Academy's Astrophysical Observatory at Potsdam, organized in collaboration with Finley-Freundlich (at St Andrews, Scotland) and with Papapetrou's and even Einstein's ${ }^{1}$ advice a campaign to observe the total Solar eclipse on 30 June 1954 from the Svedish island Öland. As for many earlier expeditions, cloudy sky prevented any observation.

Apparently, these efforts stimulated Papapetrou's research interests. Already his latest papers in Manchester were concerned with the derivation of equations of motion for spinning test particles from the conservation law $\mathcal{T}_{; v}^{\mu \nu}=0[13,37]$. Removing the restriction to test bodies leads to the question how the gravitational field of a single spinning mass would look. For the nonrotating spherical point mass Schwarzschild had given the answer, but the spinning counterpart was still an open question, at least as far as an exact solution was concerned. Solving this problem was of principle interest for tests of General Relativity in the Solar system with the rotating Sun and planets, no matter how small the effects of rotation would turn out finally.

Problems of this type had already attracted several theoreticians. As early as 1918 Lense and Thirring [35] in Vienna had calculated the exterior gravitational field of a rotating sphere, describing the influence of rotation as linear perturbation to the Schwarzschild metric. Bach [5] continued the Lense-Thirring calculation by adding terms which are quadratic in the rotation velocity. In 1924 Lanczos [33] published a simple exact solution of the matter field equations for uniformly rotating dust.

\footnotetext{
1 An exchange of letters between Naas and Einstein from November 1951 deals with the scientific chances of such a campaign [11/05/1951 Naas to Einstein (11-194.00), 11/26/1951 Einstein to Naas (11-195.00). Courtesy of the Albert Einstein Archives Jewish National and University Library Jerusalem].
} 
The matter density in his model has a minimum on the rotation axis and increases exponentially with the coordinate distance from the axis, thus compensating the increasing centrifugal forces by an increased gravitational attraction. Later papers by Andress [3] and Akeley [1,2] were mainly concerned with approximation methods for axisymmetric stationary fields. The first exact solutions of the vacuum field equations within this class of fields were found by Lewis [36] and published in an important paper in 1932. A few years later Van Stockum [46] rederived Lanczos' solution for an infinite rotating cylinder of dust particles and used one of the Lewis solutions to fit this interior field to an exterior vacuum field. A different class of exact vacuum solution of the Lewis equations was given by Papapetrou [38] soon after he arrived in Berlin. Exact solutions including time-independent gravitational fields were also systematically studied by Jordan's [26] research group at Hamburg university, mainly by Ehlers et al. [16].

However, no exact solution discovered so far could be considered as the gravitational field of a rotating nearly spherical mass. All of them had either singularities on the axis interpreted as violation of the vacuum equations and presence of a line distribution of rotating matter or, as in Papapetrou's 1953 solutions [38], had angular momentum but zero total mass, as measured by the corresponding terms in an asymptotic expansion of the metric.

From a geometrical point of view, these vacuum solutions did belong to a class of metrics, which were later shown by Papapetrou [40] as invariantly characterized by the existence of two commuting Killing fields $\xi^{\rho}$ (timelike) and $\eta^{\rho}$ (spacelike with closed orbits), which admit 2-spaces orthogonal to the group orbits. Usually this class of (vacuum) solutions is called the Lewis-Papapetrou class-also the Kerr metric belongs to this class.

The author, who had a background in astrophysics from Schwarzschild's Potsdam Observatory, entered Papapetrou's small research group in the mathematics institute in May 1959. I enjoyed the stimulating atmosphere with regular guests from East German universities and international visitors like Marie-Antoinette Tonnelat from Paris and Felix Pirani from London. My first duty was to solve a problem in Einstein's field theory with the asymmetric metric tensor. Having stood this test [14], not without help by Papapetrou, he considered me as being able to treat a more complicated problem. "Find the gravitational field of a rotating point mass as a suitable generalization of the Schwarzschild metric" was his suggestion in the late autumn of 1959. Unfortunately for the project, Papapetrou was invited to visit the Institute Henri Poincaré in Paris for 1 year. He left Berlin early in 1960, thus his valuable advice and encouragement was missed. After all, communication in that time without electronic mail was mainly confined to sending yellow post letters occasionally.

In the next sections this old attempt to tackle the problem is described. In the final section I return to the further history of the Kerr field.

\section{Lewis equations and their generalization}

The immense literature on stationary axisymmetric gravitational fields known today [45] did not exist in 1959, apart from the three basic papers by Lewis, van Stockum 
and Papapetrou. To these papers one should have added Jürgen Ehlers' 1957 thesis on exact solutions [17], but I was only later aware of this work. At some time I had access to the useful Jordan report [26], which summarized the research of the Hamburg group and included a chapter on stationary gravitational fields. Apart from these papers, the whole field was unexplored territory.

The most influential paper was that of Lewis [36]. He wrote the line element without further explanation essentially as (the notation is taken from Papapetrou [38])

$$
d s^{2}=e^{\mu}\left(d x_{1}^{2}+d x_{2}^{2}\right)+l d \phi^{2}+2 m d \phi d t-f d t^{2},
$$

where all functions $\mu, l, m$ and $f$ depend only on the two coordinates $x_{1}, x_{2}$. As shown by Lewis, with his metric the vacuum field equations $R_{\mu v}=0$ have a clear structure and admit a straightforward integration procedure: one obtains a set of three coupled nonlinear partial differential equations in two dimensions involving only the three functions $f, l, m$. Further equations allow to determine the remaining function $\mu$ by simple integration, provided a solution $f, l, m$ is given.

Lewis and Papapetrou had found special classes of solutions, but not yet one which was singular only along a single worldline and had a specific asymptotic behavior, tending to Minkowski space-time at spatial infinity.

I began to treat the problem in a systematic way. As it turned out, this was not the best method. The first question was: Is the form of the metric tensor assumed by Lewis already general enough to describe that axisymmetric vacuum field, which we wanted to find? Actually Lewis served well, but this could not be known beforehand.

We had always assumed the metric field to admit two commuting Killing vectors $\xi^{\rho}$ (timelike) and $\eta^{\rho}$ (spacelike, at least near the axis), thus $\xi_{; \sigma}^{\rho} \eta^{\sigma}-\eta_{; \sigma}^{\rho} \xi^{\sigma}=0$. One should also have asked if this assumption of an Abelian isometry group $G_{2}$ is perhaps a restriction for the problem. I do not remember our arguments for adopting commutativity (apart from Ockham's razor). Ten years later Brandon Carter [11] proved that the commutativity assumption means no loss of generality: axisymmetric stationary fields which become asymptotically flat have commuting Killing fields. More recently Alan Barnes [6] noted that the Abelian character of $G_{2}$ follows in a simple manner from the fact that the orbits of $\eta^{\rho}$ should be topologically circles. In any case, writing down normal forms for the metric in the case of a non-Abelian $G_{2}$ would have convinced us that the running coordinate along the orbits of $\eta^{\rho}$ could not be a cyclic one.

Then, assuming an Abelian $G_{2}$, it was easy to see that one can introduce new coordinates by requiring $\xi^{\rho}=\delta_{0}^{\rho}, \eta^{\rho}=\delta_{3}^{\rho}$, such that the metric depends only on the coordinates $x^{1}, x^{2}$. This special coordinate form for the Killing fields is left invariant by coordinate transformations of the type

$$
\begin{gathered}
\bar{x}^{1}=\bar{x}^{1}\left(x^{1}, x^{2}\right), \quad \bar{x}^{2}=\bar{x}^{2}\left(x^{1}, x^{2}\right), \\
\bar{x}^{3}=x^{3}+p\left(x^{1}, x^{2}\right), \quad \bar{x}^{0}=x^{0}+q\left(x^{1}, x^{2}\right),
\end{gathered}
$$

apart from linear transformations of $x^{3}, x^{0} ; \bar{x}^{1}, \bar{x}^{2}$ are invertible and $p, q$ arbitrary functions of $x^{1}, x^{2}$. 
The question was now: Is it possible to reduce the general axisymmetric stationary vacuum field to the Lewis form by means of these transformations? To my surprise, the answer turned out to be no, not in general. I tried several ways to simplify the metric. Successful was a sort of covariant reduction, where the field equations are written as three-dimensional and in a second step as two-dimensional covariant relations. Methods of this type are described in the Jordan report [26] and also in the LandauLifschitz volume "Field Theory" [34], which I just translated at that time from Russian into German. In the first step the metric tensor $g_{\mu \nu}$ was split into

$$
g_{00}=-V^{2}, \quad g_{0 i}=-\gamma_{i} V^{2}, \quad g_{i k}=\gamma_{i k}-\gamma_{i} \gamma_{k} V^{2}
$$

$(i, k=1,2,3)$. The three field equations $R_{0}^{i}=0$ then led to

$$
\left(V^{3} \kappa^{i k}\right)_{\mid k}=0
$$

where $\kappa_{i k} \equiv \frac{1}{2}\left(\gamma_{i, k}-\gamma_{k, i}\right)$, the stroke denotes the covariant derivative with respect to the 3-metric $\gamma_{i k}$ and indices are moved using $\gamma_{i k}$. If (5) holds, the quantity $E_{i k l} \kappa^{i k} V^{3}$, constructed with the three-dimensional totally antisymmetric Levi-Civita tensor $E_{i k l}$, must be a gradient $\psi_{, l}$. Solving for $\kappa_{i k}$ resulted in

$$
\kappa_{i k}=\epsilon_{i k l} \gamma^{l m} \psi_{, m} /\left(2 V^{3}\right)
$$

The condition that $\kappa_{i k}$ is a rotation, requires that $\psi$ satisfies the field equation

$$
\gamma^{k l} \psi_{, k \mid l}-3 V_{, k} \psi_{, l} \gamma^{k l} / V=0
$$

The other field equations $R_{0}^{0}=0$ and $R_{k}^{i}=0$ became

$$
\begin{gathered}
V^{3} \gamma^{k l} V_{, k \mid l}+2 \psi_{, k} \psi_{, l} \gamma^{k l}=0, \\
R_{k}^{(3) i}-\frac{1}{V} \gamma^{i l} V_{, k \mid l}+\frac{2}{V^{4}} \delta_{k}^{i} \psi_{, l} \psi_{, m} \gamma^{l m}-\frac{2}{V^{4}} \psi_{, k} \psi_{, l} \gamma^{i l}=0 .
\end{gathered}
$$

In a second step, the 3 -metric $\gamma_{i k}$ was split into two-dimensional covariant quantities (capital indices always take values 1,2 in this article):

$$
\gamma_{33}=W^{2}, \quad \gamma_{3 A}=\epsilon_{A} W^{2}, \quad \gamma_{A B}=\epsilon_{A B}+\epsilon_{A} \epsilon_{B} W^{2} .
$$

The 3-tensor equation (9) split into a scalar, vector and tensor equation in two dimensions:

$$
\begin{gathered}
\epsilon^{A B} W_{, A \| B} / W-W^{2} k^{A B} k_{A B}-2 \psi_{, A} \psi_{, B} \epsilon^{A B} / V^{4}+W_{, A} V_{, B} \epsilon^{A B} /(V W)=0, \\
\left(W^{3} k^{A B}\right)_{\| B}+W^{3} k^{A B} V_{, B} / V=0, \\
R_{B}^{(2) A}-\epsilon^{A C} W_{, B \| C} / W-2 W^{2} k^{A C} k_{B C}-\epsilon^{A C} V_{, B \| C} / V \\
+2 \delta_{B}^{A} \psi_{, C} \psi_{, D} \epsilon^{C D} / V^{4}-2 \psi_{, B} \psi, C \epsilon^{A C} / V^{4}=0 .
\end{gathered}
$$


Here

$$
k_{A B}=\frac{1}{2}\left(\epsilon_{A, B}-\epsilon_{B, A}\right)
$$

and the double stroke denotes the covariant derivative with respect to the Christoffel affinity formed with the 2-metric $\epsilon_{A B}$ (indices are moved using $\epsilon_{A B}$ ). Using the coordinate transformations (2), I assumed that the 2-metric can be transformed into a conformally flat metric:

$$
\epsilon_{A B}=\delta_{A B} e^{\mu}
$$

Equations (12) now became explicitly

$$
k_{A B, B}+3 k_{A B} W_{, B} / W+k_{A B} V_{, B} / V-k_{A B} \mu_{, B}=0 .
$$

The integration gave

$$
k_{12}=\frac{k e^{\mu}}{V W^{3}}
$$

with $k$ as integration constant. The relations (15) and (17) simplified the field equations considerably. The two equations (7) and (8) became

$$
\begin{gathered}
\Delta \psi-\left[\ln \frac{V^{3}}{W}, \psi\right]=0, \\
\Delta V+[V, W] / W+2[\psi, \psi] / V^{3}=0,
\end{gathered}
$$

where the differential operator $\Delta$ is the Laplacian in two dimensions, $\Delta=\frac{\partial^{2}}{\partial x_{1}^{2}}+\frac{\partial^{2}}{\partial x_{2}^{2}}$, and the Lewis bracket is defined as

$$
[A, B] \equiv \frac{\partial A}{\partial x^{1}} \frac{\partial B}{\partial x^{1}}+\frac{\partial A}{\partial x^{2}} \frac{\partial B}{\partial x^{2}} .
$$

Similarly, (11) is a differential equation for $W$ :

$$
\frac{\Delta W}{W}+\frac{[V, W]}{V W}-2 \frac{[\psi, \psi]}{V^{4}}=\frac{2 k^{2} e^{\mu}}{V^{2} W^{4}} .
$$

The remaining equations (13) were

$$
\begin{gathered}
\Delta \mu+\frac{\Delta W}{W}+\frac{\Delta V}{V}-2 \frac{[\psi, \psi]}{V^{4}}=-\frac{4 k^{2} e^{\mu}}{V^{2} W^{4}}, \\
\mu_{, 1}\left(\frac{V_{, 1}}{V}+\frac{W_{, 1}}{W}\right)-\mu_{, 2}\left(\frac{V_{, 2}}{V}+\frac{W_{, 2}}{W}\right)=2 \frac{\psi_{, 1}^{2}}{V^{4}}-2 \frac{\psi_{, 2}^{2}}{V^{4}}+\frac{W_{, 11}}{W}-\frac{W_{, 22}}{W}+\frac{V_{, 11}}{V}-\frac{V_{, 22}}{V},
\end{gathered}
$$




$$
\mu_{, 1}\left(\frac{V_{, 2}}{V}+\frac{W_{, 2}}{W}\right)+\mu_{, 2}\left(\frac{V_{, 1}}{V}+\frac{W_{, 1}}{W}\right)=2 \frac{V_{, 12}}{V}+2 \frac{W_{, 12}}{W}+4 \frac{\psi_{, 1} \psi_{, 2}}{V^{4}}
$$

For the function $R=V W$ one derives the simple relation

$$
R^{3} \Delta R=2 k^{2} V^{2} e^{\mu} .
$$

Apparently, Lewis was not general enough. The field equations (18, 19, 21-25) differ from the Lewis equations through the occurrence of an integration constant $k$, complicating Lewis' integration scheme (note, however, $\mu_{, 1}$ and $\mu_{, 2}$ as calculated from $(23,24)$ still satisfy $\mu_{, 1,2}=\mu_{, 2,1}$ as well as (22) in virtue of the other equations, even if $k \neq 0$ ). The complication was not the only problem. I had also carried out the reduction process inversely, splitting the metric first with regard to $\eta^{\mu}$ and then to $\xi^{\mu}$. This introduced a different set of equations with a new constant $\bar{k}$. But neither set could represent the full system of vacuum equations in the Abelian $G_{2}$ case, since both constants $k, \bar{k}$ are expected to occur. The reason why the derivation given above missed $\bar{k}$ is an implicit assumption made in the calculation of (7)-(9), that the potential $\psi$ like all other functions does not depend on $x^{3}$, i.e. $\mathcal{L}_{\eta} \psi=0$. Yet the condition $\mathcal{L}_{\eta} \psi=-2 \bar{k} \neq 0$ is compatible with the Killing symmetries for the metric and leads to the complete system of vacuum field equations in the presence of two commuting Killing fields.

Today one recognizes that the constants $k$ and $\bar{k}$ are essentially the twist scalars associated with the two Killing vectors $\eta^{\mu}, \xi^{\mu}$ :

$$
2 k=E_{\mu \nu \rho \sigma} \xi^{\mu} \eta^{v} \eta^{\rho ; \sigma}, \quad 2 \bar{k}=E_{\mu \nu \rho \sigma} \eta^{\mu} \xi^{\nu} \xi^{\rho ; \sigma} .
$$

We know that $k$ and $\bar{k}$ are constants if the vacuum equations hold, more generally, they are constants if and only if the conditions

$$
E^{\mu \nu \rho \sigma} \xi^{\lambda} R_{\lambda \mu} \xi_{\nu} \eta_{\rho}=E^{\mu \nu \rho \sigma} \eta^{\lambda} R_{\lambda \mu} \eta_{\nu} \xi_{\rho}=0
$$

are satisfied $[45,48]$.

Little is known about the existence of solutions of the generalized Lewis equations. Apparently even today no vacuum solution is known (cf. [45], see, however, [20]). Geroch [22,23] has shown that for $k, \bar{k} \neq 0$ his method of generating new vacuum solutions from given ones breaks down in the sense that the presence of Killing fields is not preserved.

I believe, Papapetrou was not happy with the extension of the Lewis equations. Indeed, his intuition turned out to be correct. He showed in a remarkable paper 6 years later [41], that the twist scalars must vanish, restoring the Lewis equations for our problem. The geometrical background of this result became clear in papers by Kundt and Trümper [32] and by Carter [10]: while the orbits of the two Killing vectors $\xi^{\mu}, \eta^{\mu}$ are always two-surface forming, the two-surface elements orthogonal to the group orbits do not fit to finite surfaces for non-vanishing twist scalars. The Lewis block diagonal form of the metric is just equivalent to "orthogonal transitivity", to the existence of two-surfaces orthogonal to the group surfaces. 
Papapetrou's 1966 result could have been found already in 1960, had the boundary conditions on the symmetry axis been analyzed: We had fairly precise ideas for the behavior of the metric at spatial infinity, but did not consider the axis, since here unknown singularities were expected. However, for a rotating localized mass the metric on part of the axis outside the body must be regular. The existence of a (at least partly) regular axis means that the cyclic Killing vector $\eta^{\mu}$ vanishes there (for a recent careful discussion of the axis conditions in axisymmetric space-times see, e.g., [43]). Since our coordinates were restricted such that $\eta^{\mu}=\delta_{0}^{\mu}$ everywhere, they must be singular on the axis. A look at (26) with the rhs now written in regular coordinates shows immediately that on the axis (and, since $k, \bar{k}$ are constants, everywhere) $k=\bar{k}=0$. If vacuum solutions with $k, \bar{k} \neq 0$ exist, they would carry singularities on the whole symmetry axis and could not represent the exterior gravitational field of a compact body. But this was not recognized in 1960.

\section{Lewis-Papapetrou class of vacuum fields}

After wasting some time with a fruitless study of the extended equations, I returned to Lewis. Letting $k=0$ in (25) gives $\Delta R=0$, thus $R$ is a harmonic function. Provided $R$ is not a constant, this allowed to introduce canonical coordinates $\rho=R$ and eliminated $W=\rho / V$ in all equations. The basic system consisted now of only two coupled nonlinear partial differential equations for the two potentials $\psi(\rho, z)$ and $V(\rho, z)$, depending on the two cylindrical coordinates $x^{1}=\rho, x^{2}=z$ :

$$
\begin{aligned}
& \Delta \psi+\frac{1}{\rho} \frac{\partial \psi}{\partial \rho}=\frac{4}{V}[V, \psi], \\
& \Delta V+\frac{1}{\rho} \frac{\partial V}{\partial \rho}=-\frac{2}{V^{3}}[\psi, \psi]+\frac{1}{V}[V, V] .
\end{aligned}
$$

One was faced with the problem to find exact solutions of this system with a prescribed behavior at spatial infinity. Lewis and Papapetrou had derived equivalent systems of equations for a different set of field quantities. No systematic integration theory was known for either system. The interesting mathematical properties of $(28,29)$ as a completely integrable system were unknown at that time. To find solutions at all, Lewis and Papapetrou had to make special ad-hoc assumptions for their potentials.

Could similar assumptions be tried for the system $(28,29)$ ? Some suggestions came from an article by Harrison [24], just published in the December 1959 issue of Physical Review. He presented many exact solutions of the vacuum field equations, obtained with heuristic methods such as separation of variables. The hope was that some of these techniques, perhaps in combination, would work also here. To have greater flexibility, I first transformed the system $(28,29)$ into a more general form, by substituting

$$
\psi=\psi\left(X^{1}, X^{2}\right), \quad V=V\left(X^{1}, X^{2}\right)
$$


assuming the $\psi, V$ are at least twice differentiable functions of $X^{A}$, with nonvanishing functional determinant

$$
D \equiv\left|\begin{array}{ll}
\frac{\partial \psi}{\partial X^{1}} & \frac{\partial \psi}{\partial X^{2}} \\
\frac{\partial V}{\partial X^{1}} & \frac{\partial V}{\partial X^{2}}
\end{array}\right| \neq 0
$$

Then the inverse functions $X^{A}=X^{A}(\psi, V)$ exist. Introducing (30) into $(28,29)$, one obtains equations of the type (summation convention for repeated indices)

$$
\Delta X^{A}+\frac{1}{\rho} \frac{\partial X^{A}}{\partial \rho}+\lambda_{B C}^{A}\left[X^{B}, X^{C}\right]=0,
$$

where the six quantities $\lambda_{B C}^{A}=\lambda_{C B}^{A}$ are functions of the new independent field quantities $X^{A}$. I observed that (32) is invariant with respect to substitutions

$$
\bar{X}^{A}=\bar{X}^{A}\left(X^{B}\right)
$$

provided the $\lambda_{B C}^{A}$ transform as an affine connection (considering the $X^{A}$ as independent variables):

$$
\bar{\lambda}_{B C}^{A}=\frac{\partial \bar{X}^{A}}{\partial X^{I}}\left(\frac{\partial^{2} X^{I}}{\partial \bar{X}^{B} \partial \bar{X}^{C}}+\lambda_{D E}^{I} \frac{\partial X^{D}}{\partial \bar{X}^{B}} \frac{\partial X^{E}}{\partial \bar{X}^{C}}\right) .
$$

Was there any hope to reduce $(28,29)$ to linear equations using a suitable nonlinear transformation (33)? A necessary condition is the vanishing of the Ricci tensor $\mathcal{R}_{A B}=$ $\lambda_{A B, C}^{C}-\lambda_{C A, B}^{C}+\lambda_{C D}^{C} \lambda_{A B}^{D}-\lambda_{D A}^{C} \lambda_{C B}^{D}$, formed with the $\lambda$-connection in the twodimensional space of potentials $\left(X^{1}, X^{2}\right)$. A short calculation had shown that already $\mathcal{R}_{11}=-3 / V^{2}$ is nonzero, the nonlinearity could not be removed.

The simple potential space formalism allowed to answer also other questions. The integration idea was to try heuristic methods for other potentials, if the original set $(V, \psi)$ failed. At least for Papapetrou's basic assumption in [38] this hope had to be given up. In my notation his condition is

$$
V_{, 1} \psi_{, 2}-V_{, 2} \psi_{, 1}=0
$$

This relation is invariant with respect to arbitrary coordinate transformations $x^{\prime A}=$ $x^{\prime A}\left(x^{B}\right)$, but it is also invariant with respect to arbitrary substitutions (33) of the potentials. Thus choosing other potentials does not increase the chance to find solutions beyond the special Papapetrou class. Another heuristic assumption, introduced by Lewis for a pair of his variables, was $X^{1}=p\left(X^{2}\right)$, a functional relationship between two potentials. It is seen that (35) is satisfied in this case, hence also this restriction leads only to solutions within the special Papapetrou class. 
An important restriction for the solutions is the proper behavior at spatial infinity, assumed as $\left(r=\sqrt{\rho^{2}+z^{2}}\right)$

$$
f \rightarrow 1, l \rightarrow \rho^{2}, m \rightarrow \lambda \frac{\rho^{2}}{r^{3}}
$$

for the Papapetrou functions $f, l, m$, where $\lambda$ is proportional to the angular momentum ("strong boundary condition"). For the potential $\psi$ this transforms to $\psi \rightarrow \lambda z /\left(2 r^{3}\right)$. The strong boundary condition ensures that the metric tends to the Minkowski spacetime at spatial infinity $r \rightarrow \infty$, it also provides finite values for the total angular momentum [15]. Unfortunately, metrics of the special Papapetrou class which satisfy the strong boundary condition have zero total mass or energy, this follows immediately from an $1 / r$ expansion of $(28,29)$ and $(35)$.

\section{Sample solutions}

Vacuum metrics with $V W=$ const did not allow canonical coordinates, but satisfy simple equations. One obtains from $(18,19,21-25)$ with $k=0$ the compatible set

$$
\Delta \psi=0, \quad \Delta \mu=0, \quad\left(V^{2}\right)_{, 1}=2 \epsilon \psi_{, 2}, \quad\left(V^{2}\right)_{, 2}=-2 \epsilon \psi_{, 1}
$$

$\left(\epsilon^{2}=1\right) . \psi$ and $\mu$ are harmonic functions, their singularities had to be considered as resulting from a singular matter distribution.

It seems obvious here to interpret $x^{1}, x^{2}$ as quasi-Cartesian coordinates in a plane orthogonal to the $x^{3}$-axis. The Killing vector $\eta^{\mu}$ then represents a translational symmetry along the $x^{3}$-axis. The simplest solutions have a singularity at the origin of $x^{1}, x^{2}$ and were believed to describe the exterior gravitational field of an infinite rotating cylinder along the $x^{3}$-axis. But such fields had nothing to do with the gravitational field of a rotating point mass. Later Hoffman [25] discussed this class of stationary fields.

Harrison's separation technique as applied to (32) was my main working tool. Similar methods are still used today [21]. In principle, this technique can be applied not only to transformed potentials but also in the case of transformed coordinates. Thus I filled many sheets of paper with formulae of that type, too often stopping the calculation once it became clear that the required strong boundary conditions could not be satisfied.

For example, in the case of quasi-spherical coordinates $\rho=r \sin \theta, z=r \cos \theta$ one has with $f=V^{2}$

$$
\begin{gathered}
\psi_{, r r}+\frac{2}{r} \psi_{, r}+\frac{\psi_{, \theta \theta}}{r^{2}}+\cot \theta \frac{\psi_{, \theta}}{r^{2}}=2 \frac{f_{, r}}{f} \psi_{, r}+\frac{2}{r^{2}} \frac{f_{, \theta}}{f} \psi_{, \theta}, \\
f_{, r r}+\frac{2}{r} f_{, r}-\frac{1}{f} f_{, r}^{2}+\frac{1}{r^{2}} f_{, \theta \theta}+\cot \theta \frac{f_{, \theta}}{r^{2}}-\frac{1}{r^{2}} \frac{f_{, \theta}^{2}}{f}=-\frac{4}{f} \psi_{, r}^{2}-\frac{4}{r^{2} f} \psi_{, \theta}^{2} .
\end{gathered}
$$


Separation assumptions led to a number of subcases. In the case where both $\psi$ and $f$ depend only on the radial coordinate $r$, (38) gives $\psi_{, r}=c f^{2} / r^{2}, c=$ const. Introducing this into (39) leads to an equation for $f$ alone:

$$
f_{, r r}+\frac{2}{r} f_{, r}-\frac{1}{f} f_{, r}^{2}+4 \frac{c^{2}}{r^{4}} f^{3}=0 .
$$

The solutions are (Gothic letters denote hyperbolic functions)

$$
-g_{00}=V^{2}=\frac{\alpha}{\mathfrak{C o s}(\gamma+\beta / r)}, \quad \psi=-\frac{\alpha}{2} \mathfrak{T a n}(\gamma+\beta / r) .
$$

The three integration constants $\alpha, \beta, \gamma(c=\beta /(2 \alpha))$ are not independent, requiring $\lim g_{00} \rightarrow-1$ for $r \rightarrow \infty$ gives $\alpha=\mathfrak{C o s} \gamma$.

Also the equations for $\mu$ can be integrated, the further non-vanishing components of the metric are

$$
\begin{aligned}
g_{r r} & =\frac{1}{\alpha} \operatorname{Cos}(\gamma+\beta / r) e^{-\beta^{2} \sin ^{2} \theta /\left(4 r^{2}\right)}, \\
g_{\theta \theta} & =r^{2} g_{r r}, \\
g_{\phi \phi} & =\left(\operatorname{Cos}(\gamma+\beta / r) / \alpha-\frac{\beta^{2} \cot ^{2} \theta}{\alpha r^{2} \operatorname{Cos}(\beta / r+\gamma)}\right) r^{2} \sin ^{2} \theta, \\
g_{\phi t} & =\frac{\beta \cos \theta}{2 \operatorname{Cos}(\gamma+\beta / r)} .
\end{aligned}
$$

Expansion of $g_{00}$ in reciprocal powers of $r$ shows that $2 M=\beta \mathfrak{T a n} \gamma$ is the coefficient of the $1 / r$-term. Several arguments suggest that $M$ is the total energy of the field. In 1960 I used pseudotensors derived from the Lagrange density of the gravitational field according to Noether's procedure [15], but one obtains the same result with the ADM mass formula [4]. Again, however, this was clearly not the solution we had looked for, since the boundary conditions at spatial infinity are not satisfied for the nondiagonal term, $\psi$ does not vanish but tends to the constant $-\alpha \mathfrak{T a n} \gamma / 2$ for $r \rightarrow \infty$. Thus the total angular momentum [15] diverges. Since $\psi, V$ depend on $r$ only, a short look shows that (35) is satisfied, thus the solution belongs to the special Papapetrou class. The metric has nonzero total energy only because the strong boundary conditions are violated.

It was time consuming and unsatisfactory to search for solutions with the rather simple trial-and-error methods at hand. According to my 1960 notebook, I was impressed by Buchdahl's procedure to obtain new stationary solutions from a given static or stationary vacuum field. Later many successful recipes were developed to realize this idea of solution generation [27,45], starting from the pioneering papers by Buchdahl [8,9] and by Ehlers [18]. This has finally opened the door to the solution space. 


\section{Any chance in 1960?}

But still I did not give up. On March 6, 1960, I sent Papapetrou a letter with a short summary of results obtained so far (translated from German, signature of metric changed):

[...] After two weeks of skiing with best snow conditions I'm back to Berlin. The state of my work is roughly as follows.

(1) It was guessed that forming a normal form for $g_{\mu \nu}$ already before the field equations are reduced is preferable, since more transformation freedom is available. However, Petrov's choice $\left(g_{11}=1, g_{12}=g_{13}=g_{10}=0\right)$ offers no advantage to my previous approach for a concrete solution of the field equations. It stands for a certain choice of coordinates in the $x_{1}-x_{2}$-space, but the field equations which must be solved next are not simplified.

(2) The reverse reduction method (first $x_{3}$ then $x_{0}$ ) gives very complicated equations as in the previous case, if $\bar{k} \neq 0$. $\left(\bar{k}=0\right.$ now means $g_{0 I}=g_{I 3} g_{03} / g_{33}$; for the original reduction sequence the analogous condition $k=0$ was $g_{3 I}=$ $\left.g_{0 I} g_{03} / g_{00}\right)$. But if we assume $\bar{k}=0$ and require Minkowskian boundary conditions, the equations reduce to those of Lewis.

(3) A class of solutions $(k=0)$, which are presumably uninteresting physically, obviously describe the gravitational field of a rotating cylinder with a multipole matter source. These fields are independent of $z$, but they lost rotational symmetry, depending on the polar coordinates in a plane orthogonal to the cylinder axis. A closer inspection seems not to be worthwhile.

(4) My earlier solution

$$
\begin{aligned}
d s^{2}= & -\frac{\alpha}{\operatorname{Cos}(\beta / r+\gamma)} d t^{2}+\frac{\mathfrak{C o s}(\beta / r+\gamma)}{\alpha} e^{-\beta^{2} \sin ^{2} \theta /\left(4 r^{2}\right)}\left(d r^{2}+r^{2} d \theta^{2}\right) \\
& +\left(\frac{\operatorname{Cos}(\beta / r+\gamma)}{\alpha}-\frac{\beta^{2} \cot ^{2} \theta}{\alpha r^{2} \mathfrak{C o s}(\beta / r+\gamma)}\right) r^{2} \sin ^{2} \theta d \phi^{2} \\
& +\frac{\beta \cos \theta}{\mathfrak{C o s}(\beta / r+\gamma)} d \phi d t
\end{aligned}
$$

has some interesting properties which might render it acceptable in spite of the missing boundary condition for $g_{03}$ :

(a) The total energy (according to Møller) is finite and depends as in the Schwarzschild case on the $1 / r$ term in the expansion of $g_{00}$.

(b) The total momentum vanishes.

(c) For $\alpha \rightarrow \infty$ and neglecting the terms with $1 / r^{2}$ the solution tends asymptotically to the Schwarzschild solution.

(d) For $r \rightarrow \infty$ the space becomes homogeneous, but is no longer isotropic. Since there exists a distinguished direction, this appears to be reasonable.

Mr. Treder and I agree that this type of solution (the given one is only the simplest) should be considered as physically reasonable. I would like to ask you for your opinion. 
(5) Presently I am trying to transform the field equations with $k=0$ using suitable coordinate conditions in the $x_{1}-x_{2}$-space to give them a convenient structure. I hope that within one of these coordinate systems the solution which we are looking for takes a fairly simple form, and can therefore be found relatively easily. But one must be lucky!

In spite of the small success so far I still believe that one can find a stationary solution which satisfies all requirements.

It would have been interesting to know Papapetrou's reaction, but I do not remember having obtained a response.

Needless to say, I had no luck with the recipe proposed under item (5). But the recipe itself-looking for suitable coordinates in the $x^{1}-x^{2}$-space-was indeed a route to the Holy Grail, the rotating Schwarzschild field: transforming the cylindrical coordinates $\rho, z$ into some kind of radial and angular coordinates $r, \theta$ by means of

$$
\rho=\sqrt{r^{2}+a^{2}-2 r M \sin \theta}, \quad z=(r-M) \cos \theta,
$$

as done by Ernst [19] after Kerr's discovery, leads to the Kerr solution with the potentials

$$
V^{2}=1-\frac{2 r M}{r^{2}+a^{2} \cos ^{2} \theta}, \quad \psi=-\frac{a M \cos \theta}{r^{2}+a^{2} \cos ^{2} \theta} .
$$

This looks simple indeed. But how could one have figured out the coordinate transformation (46) in 1960? There exist other coordinates in which the Kerr functions appear fairly simple, e.g. spheroidal prolate coordinates [7]. To find them by trial-and-error would not have been easy, but it was not impossible, given sufficient diligence and persistence.

In 1968 Ernst [19] also found that the complex combination $\mathcal{E}=V^{2}+2 i \psi$ satisfies an elegant differential equation, easily derivable from $(28,29)$, which has dominated the research on stationary axisymmetric gravitational fields since that time. The Kerr solution of the complex Ernst equation has the simple form $\mathcal{E}=1-2 M /(r-i a \cos \theta)$ in the coordinates $r, \theta$ given by (46). The related function $\xi=(1+\mathcal{E}) /(1-\mathcal{E})$ (essentially a potential transformation as discussed above) satisfies a similar differential equation. Kerr is then represented by $\xi=r / M-i a \cos \theta / 1-1$. Evidently, this is now even an almost trivial solution of the differential equation for $\xi$. A solution could hardly be simpler.

It is satisfying that both concepts, coordinate transformation in $x^{1}-x^{2}$-space and potential transformation, were finally so successful, thanks to the efforts by F.J. Ernst.

\section{The winner is Kerr}

The slow progress as well as Papapetrou's absence from Berlin rapidly diminished the amount of time I spent in 1960 for the rotating Schwarzschild problem. During the year I became interested in many other questions of gravitation. Not being guided by my 
boss, I worked on boundary conditions, surface layers, shock waves and other problems of gravitational radiation. In particular, the characteristic initial value problem for the Einstein field equations was a very interesting topic, since here one could handle the two intrinsic degrees of freedom of the gravitational field rather directly. When Papapetrou returned from Paris in the beginning of 1961, I confronted him with new ideas about these perhaps more actual problems. There was a rumor that Bondi and his group in London were working on similar questions. The problem of stationary fields was forgotten for the present, at least for me.

In the meantime the political situation had changed for the worse. There was the danger that Papapetrou's position as prominent scientist in a communist country could lead to problems for his relatives in Greece. The Cold War was present everywhere, particularly in the divided city of Berlin, whose borders were still open. A steadily increasing number of East Germans escaped to the West. Suddenly, on 13 August 1961, the borders were closed by the communist authorities. Quite unexpectedly, the Berlin Wall was built, cutting off the free Western part of Berlin from the surrounding East German territory. For Papapetrou and his wife the year in Paris was so pleasing compared with the difficult situation in East Berlin, that they decided-weeks before the borders were closed-to stay permanently in Paris. Not being citizens of the East German state, they were allowed to go.

This was unpleasant news. I had to finish my $\mathrm{PhD}$ thesis on wave solutions and the characteristic initial value problem sufficiently early in 1961 that Papapetrou could act as adviser, before he finally left East Berlin at the end of the year. Apparently, no time was left to discuss rotating metrics in depth.

While I did not seriously return to the spinning body, Papapetrou never forgot the problem. In at least two papers $[39,40]$ he further explored the properties of axisymmetric metrics and found a new subclass of solutions. However, also these metrics did not satisfy the required boundary conditions at infinity.

Also other groups had no luck. In their well-known survey on exact solutions published in the legendary Written volume in 1962, Ehlers and Kundt [16] had to admit that "the old problem of constructing rigorously the field of a finite rotating body is as yet unsolved, even as to its exterior part".

The solution of the long-standing problem came 1963 from the New Zealander Roy P. Kerr in a different way. Kerr received his PhD 1959 from Cambridge University (MA), worked later at Syracuse University and with the US Air Force relativity group under Joshua Goldberg at Wright-Patterson Field in Ohio, before he came to the University of Texas at Austin in the academic year 1962/1963. Here, in a newly founded Center of Relativity, organized by Alfred Schild, a circle of relativists had gathered, including besides Schild and Kerr temporarily also Roger Penrose, Ray Sachs, Engelbert Schücking and other excellent scientists. In a recent article Kerr gave a detailed description of his discovery in this stimulating environment [30]. He used a kind of null tetrad formalism, assuming from the beginning an algebraically special space-time. Both the Schwarzschild metric as well as the Kerr metric are of Petrov type D, thus this restriction was crucial. In rather complicated calculations (more complicated than I ever tried) he further restricted the fields to satisfy stationary and then axisymmetric symmetries, so he finally found the famous solution bearing his name. His two-page paper "Gravitational Field of a Spinning Mass as an Example 
of Algebraically Special Metrics" was published in the 1 September 1963 issue of Physical Review Letters.

Kerr presented his solution at the First Texas Symposium on Relativistic Astrophysics held in Dallas in December 1963 [28]. Papapetrou was possibly not aware of Kerr's article when he came to the Texas symposium, since the note [40] was presented by Louis de Broglie on December 4 (Séance du 4 décembre), apparently before his departure to the States: therein is no reference to Kerr's paper. Kip Thorne has given a vivid description of the Texas meeting in his book [47]:

To foster dialogue between the relativists and the astronomers and astrophysicists, and to catalyze progress in the study of quasars, a conference of three hundred scientists was held on 16-18 December 1963, in Dallas, Texas [...] Lectures went on almost continuously from 8:30 in the morning until 6 in the evening with an hour out for lunch, plus 6 P.M. until typically 2 A.M. for informal discussions and arguments. Slipped in among the lectures was a short, 10-min presentation by a young New Zealander mathematician, Roy Kerr, who was unknown to the other participants. Kerr had just discovered his solution of the Einstein field equation - the solution which, one decade later, would turn out to describe all properties of spinning black holes, including their storage and release of rotational energy [...]; the solution which [...] would ultimately become a foundation for explaining the quasars' energy. However, in 1963 Kerr's solution seemed to most scientists only a mathematical curiosity; nobody even knew it described a black hole — though Kerr speculated it might somehow give insight into the implosion of rotating stars.

The astronomers and astrophysicists had come to Dallas to discuss quasars; they were not at all interested in Kerr's esoteric mathematical topic. So, as Kerr got up to speak, many slipped out of the lecture hall and into the foyer to argue with each other about their favorite theories of quasars. Others, less polite, remained seated in the hall and argued in whispers. Many of the rest catnapped in a fruitless effort to remedy their sleep deficits from the late-night science. Only a handful of relativists listened, with rapt attention.

This was more than Achilles Papapetrou, one of the world's leading relativists, could stand. As Kerr finished, Papapetrou demanded the floor, stood up, and with deep feeling explained the importance of Kerr's feat. He, Papapetrou, had been trying for 30 years to find such a solution of Einstein's equation, and had failed, as had many other relativists. The astronomers and astrophysicists nodded politely, and then, as the next speaker began to hold forth on a theory of quasars, they refocused their attention, and the meeting picked up pace.

Kerr's paper of 1963 is a masterpiece of clarity and conciseness and, as Chandrasekhar noted, is "surprisingly complete in enumerating the essential features of the solution" [12]. It has only one deficiency, it gives few hints how the solution was derived. When Wolfgang Kundt came to East Berlin in the spring of 1964 for a visit, we discussed Kerr's metric, but found it hard to verify that it is indeed a solution of the vacuum equations. Many of our colleagues had the same problem. A derivation was published 
by Kerr and Schild in 1965 [29], and a more easily accessible exposition of this derivation can be found in [42].

Summarizing, one can shortly answer why the pre-Kerr approaches failed: we had the adequate differential equations, essentially identical to the Ernst equation split into real and imaginary parts. Yet the group-theoretical properties of the solutions were not recognized, and thus no proper key to the unexpectedly large solution space was found.

Kerr's new way circumvented the problem by a restriction to algebraically special metrics from the beginning, a condition which could not easily be expressed in the formalism we had used. In spite of more complicated equations he finally brilliantly succeeded, not least because of his persistence.

Acknowledgments The author is grateful to R. Kerr, W. Kundt, A. Rendall and G. Wallis for reading the manuscript and for comments and suggestions. He also thanks the referees for suggesting improvements.

Open Access This article is distributed under the terms of the Creative Commons Attribution Noncommercial License which permits any noncommercial use, distribution, and reproduction in any medium, provided the original author(s) and source are credited.

\section{References}

1. Akeley, E.S.: The axially symmetric stationary gravitational field. Phil. Mag. (Lond.) 11, 322-330 (1931)

2. Akeley, E.S.: The rotating fluid in the relativity theory. Phil. Mag. (Lond.) 11, 330-344 (1931)

3. Andress, W.R.: Some solutions of Einstein's gravitational equations for systems with axial symmetry. Proc. R. Soc. (Lond.) A126, 592-602 (1933)

4. Arnowitt, R., Deser, S., Misner, C.W.: Coordinate invariance and energy expressions in general relativity. Phys. Rev. 167, 997-1006 (1961)

5. Bach, R.: Neue Lösungen der Einsteinschen Gravitationsgleichungen. A. Das Feld in der Umgebung eines langsam rotierenden kugelähnlichen Körpers von beliebiger Masse in 1. und 2. Annäherung. Math. Z. 13, 119-133 (1922)

6. Barnes, A.: Some restrictions on the symmetry groups of axially symmmetric space-times. Class. Quant. Grav. 18, 5511-5520 (2001)

7. Bergamini, R., Viaggiu, S.: A novel derivation for Kerr metric in Papapetrou gauge. Class. Quant. Grav. 21, 4567-4573 (2004)

8. Buchdahl, H.A.: Reciprocal static solutions of the equations of the gravitational field. Aust. J. Phys. 9, 13-18 (1956)

9. Buchdahl, H.A.: Reciprocal static metrics and scalar fields in the general theory of relativity. Phys. Rev. 115, 1325-1328 (1959)

10. Carter, B.: Killing horizons and orthogonally transitive groups in space-time. J. Math. Phys. 10, 70-81 (1969)

11. Carter, B.: The commutation property of a stationary, axisymmetric system. Commun. Math. Phys. 17, 233-238 (1970)

12. Chandrasekhar, S.: The Mathematical Theory of Black Holes. Oxford University Press, New York (1983)

13. Corinaldesi, E., Papapetrou, A.: Spinning test particles in general relativity: II. Proc. R. Soc. A 64, 259-268 (1952)

14. Dautcourt, G.: Sur la solution de l'équation d'Einstein $g_{\mu_{+} v_{-} ; \rho}=0$. C. R. Acad. Sci. Paris 249, 2159-2161 (1959)

15. Dautcourt, G.: Energie, Impuls und Drehimpuls in der allgemeinen Relativitätstheorie. Ann. Phys. (Leipz.) 9, 309-317 (1961)

16. Ehlers, J., Kundt, W.: Exact solutions of the gravitational field equations. In: Witten, L. (ed.) Gravitation: An Introduction to Current Research, pp. 49-101. Wiley, New York (1962) 
17. Ehlers, J.: Konstruktionen und Charakterisierung von Lösungen der Einsteinschen Gravitationsfeldgleichungen. Dissertation Hamburg (1957)

18. Ehlers, J.: Transformations of static exterior solutions of Einstein's gravitational field equations into different solutions by means of conformal mappings. Colloques Int. C.N.R.S. Les théories relativistes de la gravitation, vol. 91, p. 275 (1961)

19. Ernst, F.J.: New formulation of the axially symmetric gravitational field problem. Phys. Rev. 167, 1175-1178 (1968)

20. Gaffet, B.: The Einstein equations with two commuting Killing vectors. Class. Quant. Grav. 7, 2017-2044 (1990)

21. Gariel, J., Marcilhacy, G., Santos, N.O.: Stationary axisymmetric solutions involving a third order equation irreducible to Painlevé transcendents. J. Math. Phys. 49, 022501 (2008)

22. Geroch, R.: A method for generating solutions of Einstein's equations. J. Math. Phys. 12, 918-924 (1970)

23. Geroch, R.: A method for generating solutions of Einstein's equation: II. J. Math. Phys. 13, 394-404 (1970)

24. Harrison, B.K.: Exact three-variable solutions of the field equations of general relativity. Phys. Rev. 116, 1285-1296 (1959)

25. Hoffman, R.B.: Stationary "non-canonical" solutions of the Einstein vacuum field equations. J. Math. Phys. 10, 953-956 (1969)

26. Jordan, P.: Research on the theory of general relativity. Mimeographed Notes, vol. 61(514) 1233. Air Research and Development Command, European Office, Air Force Contract AF (1958)

27. Kerr, R.P.: Gravitational field of a spinning mass as an example of algebraically special metrics. Phys. Rev. Lett. 11, 237-238 (1963)

28. Kerr, R.P.: Gravitational collapse and rotation. In: Robinson, I., Schild, A., Schucking, E.L. (eds.) Quasi-Stellar Sources and Gravitational Collapse, pp. 99-102. University of Chicago Press, Chicago (1965)

29. Kerr, R.P., Schild, A.: A new class of vacuum solutions of the Einstein field equations. In: Atti del Convegno Sulla Relativita Generale: Problemi dell'Energia e Onde Gravitazionali. G. Barbera, Florence (1965)

30. Kerr, R.P.: Discovering the Kerr und Kerr-Schild metrics. arXiv0706.1109v1 [gr-qc]

31. Kordas, P.: Aspects of solution-generatic techniques for space-times with two commuting killing vectors. Class. Quant. Grav. 31, 1941-1984 (1998)

32. Kundt, W., Trümper, M.: Orthogonal decomposition of axi-symmetric stationary space-times. Z. Phys. 192, 419-422 (1966)

33. Lanczos, K.: Über eine stationäre Kosmologie im Sinne der Einsteinschen Gravitationstheorie. Z. Phys. 21 (1924) 73-110 [reprinted as Golden Oldie. Gen. Relativ. Grav. 29(3), 363 (1997)]

34. Landau, L.D., Lifschitz, E.M.: Klassische Feldtheorie. Akademie-Verlag, Berlin (1963)

35. Lense, J., Thirring, H.: Über den Einfluß der Eigenrotation auf die Bewegung der Planeten und Monde nach der Einsteinschen Gravitationstheorie. Phys. Z. 19 156-163 (1918) [reprinted as Golden Oldie. Gen. Relativ. Grav. 16(8), 727-741 (1984)]

36. Lewis, T.: Some special solutions of the equations of axially symmetric gravitational fields. Proc. R. Soc. Lond. A136, 176-192 (1932)

37. Papapetrou, A.: Spinning test particles in general relativity: I. Proc. R. Soc. A 64, 248-258 (1952)

38. Papapetrou, A.: Eine rotationssymmetrische Lösung in der allgemeinen Relativitätstheorie. Ann. Phys. (Leipz.) 12, 309-315 (1953)

39. Papapetrou, A.: Quelques remarques sur les champs gravitationnels stationnaires. C. R. Acad. Sci. Paris 257, 2797-2800 (1963)

40. Papapetrou, A.: Champs gravitationnels stationnaires à symétrie axiale. C. R. Acad. Sci. Paris 285, 90-93 (1964)

41. Papapetrou, A.: Champs gravitationnels stationnaires à symétrie axiale. Ann. Inst. Henri Poincare IV, 83-105 (1966)

42. Plebanski, J., Krasinski, A.: An Introduction to General Relativity and Cosmology, Chap. 20. Cambridge University Press, London (2006)

43. Rinne, O., Stewart, J.M.: A strongly hyperbolic and regular reduction of Einstein's equations for axisymmetric space-times. Class. Quant. Grav. 22, 1143-1166 (2005)

44. Schwarzschild, K.: Über das Gravitationsfeld eines Massenpunktes nach der Einsteinschen Theorie. Sitz. Preuss. Akad. Wiss. 189 (1916) 
45. Stephani, H., Kramer, D., MacCallum, M., Hoenselaers, C., Herlt, E.: Exact Solutions of Einstein's Field Equations, 2nd edn. Cambridge University Press, London (2003)

46. van Stockum, W.J.: The gravitational field of a distribution of particles rotating about an axis of symmetry. Proc. R. Soc. Edinb. 57, 135-154 (1937)

47. Thorne, K.S.: Black holes and time warps. Foreword by Stephen Hawking, pp. 341-342. W.W.Norton \& Company, New York (2004)

48. Wald, R.M.: General Relativity. The University of Chicago Press, Chicago (1984) 